\title{
The changing pattern of methicillin-resistant Staphylococcus aureus clones in Latin America: implications for clinical practice in the region
}

\begin{abstract}
Methicillin-resistant Staphylococcus aureus (MRSA) clones belonging to the Brazilian, Pediatric, Cordobes/Chilean and New York/Japan clonal complexes are widely distributed across Latin Ameri$\mathrm{ca}$, although their individual distribution patterns and resistance to antimicrobial drugs are constantly changing. Furthermore, clones with increased virulence are beginning to appear more frequently both in hospital and community settings, and there is evidence that virulence factors can be transferred between hospital- and community-associated clones through recombination. These changing patterns have significant implications for clinical practice in the region. Most importantly, clinicians need to be aware of the changing antimicrobial resistance profile of circulating MRSA clones in their region in order to choose the most appropriate empiric antimicrobial therapy. Thus, regional molecular epidemiology programs are required across the region to provide accurate identification and characterization of circulating MRSA clones.
\end{abstract}

Keywords: MRSA, clones, molecular epidemiology, Latin America.
Authors

Eduardo RodríguezNoriega $^{1}$

Carlos Seas ${ }^{2}$

on behalf of the Latin

American Working

Group on Gram

Positive Resistance.

${ }^{1}$ Hospital Civil

de Guadalajara,

Universidad de

Guadalajara, Jalisco,

Mexico.

${ }^{2}$ Universidad

Peruana Cayetano

Heredia, Lima, Peru.

\section{INTRODUCTION}

Methicillin-resistant Staphylococcus aureus (MRSA) poses a major threat to public health worldwide, due to the rapid spread and diversification of pandemic MRSA clones with increasing virulence and antimicrobial resistance. In Latin America, MRSA is a leading cause of nosocomial infections, and the prevalence of MRSA in community-acquired infections is growing. ${ }^{1}$

Although relatively few studies have addressed the molecular epidemiology of MRSA clones across Latin America, it is clear that several clones circulate in the region and that these differ in their virulence, antimicrobial resistance profile and geographical distribution. ${ }^{1,2}$ Characterization of these clones is important if appropriate local treatment strategies are to be developed. For example, a thorough knowledge of clones circulating within a region may be used to assess the relationship between clonal types, disease symptoms, antibiotic choice and clinical outcomes. Furthermore, understanding why specific clones predominate in different regions of Latin America is an important and necessary step towards developing the most effective strategies for controlling the spread of MRSA in the region.

Here, we summarize current understanding of the spread of pandemic MRSA clones and highlight the distribution of major clones across Latin America, both in hospitals and in the community. Specific virulence factors and bacterial resistance patterns are highlighted, and their impact on clinical outcome is discussed.

\section{EVOLUTION OF MRSA CLONES}

\section{Evolution of bacterial clones}

Bacterial clones are genetically identical cells descended from a single common ancestor. Over time, members of a single clone may differentiate through point mutations, recombination, and the acquisition or deletion of mobile genetic elements. This differentiation provides additional means for the acquisition of pathogenic charac-
Correspondence to: Dr Eduardo Rodríguez-Noriega Hospital Civil de Guadalajara Fray Antonio Alcalde Instituto de Patología Infecciosa y Experimental Centro Universitario Ciencias de la Salud Universidad de Guadalajara Jalisco, México Hospital 308, Colonia El Retiro C.P. 44280 , Guadalajara Jalisco, México Phone: +52-33-36145568

Fax: +52-33-36850501

E-mail: idfcolima@ yahoo.com 
teristics, such as antibiotic resistance. Thus, genetic variation gives rise to extensive genomic and phenotypic diversity.

\section{Emergence of antibiotic resistant $S$. aureus clones}

Clones of $S$. aureus have a history of antibiotic resistance that began within 4 years of the introduction of penicillin into clinical practice, ${ }^{3}$ and by 1944 , clones of S. aureus resistant to penicillin had been isolated. In the subsequent years, S. aureus became resistant to all of the natural penicillins.

MRSA was first reported in the early 1960s, shortly after the introduction of methicillin. ${ }^{4}$ Early MRSA clones had similar genetic properties to the methicillin-susceptible $S$. aureus (MSSA) clones that were epidemic in Europe. ${ }^{5}$ MRSA exhibits resistance to methicillin through a penicillin-binding protein encoded by the gene mecA, which was acquired by successful clones of MSSA from an unknown heterologous source. The mecA gene is carried by a mobile genetic element called the staphylococcal cassette chromosome mec (SCCmec). Multiple forms of SCCmec have arisen through the horizontal transfer of $m e c A$ in independent events, and, to date, seven main forms have been identified (I, II, III, IV, V, VI and VII). ${ }^{6}$ All types of SCCmec confer resistance to -lactam antibiotics, and SCCmec types II and III provide resistance to multiple classes of antibiotics. ${ }^{7}$

During the evolution of MRSA clones, independent excision of SCCmec is a common phenomenon, resulting in the loss of methicillin-resistance and the transformation of a MRSA clone into a MSSA clone. Hence, clones may evolve from MSSA into MRSA, or from MRSA into MSSA, through the acquisition and excision of SCCmec, respectively. ${ }^{8}$
Several molecular typing methods are used routinely to characterize MRSA clones, including pulsed-field gel electrophoresis (PFGE), multilocus sequence typing (MLST) and SCCmec typing. ${ }^{9}$ These methods have helped researchers to map the spread and evolutionary path of MRSA clones. ${ }^{7,8}$

MRSA has traditionally been regarded as a nosocomial pathogen, ${ }^{10}$ but more recently, MRSA infections have appeared in community settings. ${ }^{11}$ The clones typically responsible for hospital- and community-acquired MRSA infections have been classified as healthcare-associated MRSA (HA-MRSA) and community-associated MRSA (CA-MRSA), respectively. ${ }^{12}$ These clones can be distinguished based on specific microbiologic and genetic characteristics, and often have different epidemiologic, clinical and therapeutic characteristics (Table 1). ${ }^{10,11}$ Occasionally, hospital-acquired infections may be derived from CA-MRSA strains, and infections acquired in the community may carry healthcareassociated risk factors. Definitive HA-MRSA and CA-MRSA designations for individual clones, therefore, rely on microbiologic and genetic characterization, and the terms 'healthcare-acquired' and 'community-acquired' refer to the location at which the infection was acquired. ${ }^{12}$

\section{International spread of MRSA clones}

S. aureus clones spread quickly around the world, ${ }^{13}$ disseminating efficiently between countries, within countries and in smaller geographic areas, and usually with concomitant evolution from a methicillin-sensitive to a methicillin-resistant phenotype. ${ }^{5,14}$ Most nosocomial MRSA infections worldwide are derived from one of five major lineages known

Table 1. Common characteristics of infections caused by HA-MRSA and CA-MRSA

\begin{tabular}{|c|c|c|}
\hline Characteristic & HA-MRSA & CA-MRSA \\
\hline Year of discovery & 1961 & $1980 \mathrm{~s}$ \\
\hline Population at risk & $\begin{array}{l}\text { Patients having previous hospitalization, } \\
\text { surgery, residence in long-term care } \\
\text { facilities, dialysis, permanent indwelling } \\
\text { catheters, intensive care unit }\end{array}$ & $\begin{array}{l}\text { Children, homeless, men who have sex } \\
\text { with men, athletes, military recruits, } \\
\text { jail inmates, native Americans, Pacific } \\
\text { Islanders, adult emergency department } \\
\text { patients }\end{array}$ \\
\hline Main clinical syndromes & $\begin{array}{l}\text { Bacteremia, HAP, VAP, catheter- and } \\
\text { prosthetic-related infections }\end{array}$ & $\begin{array}{l}\text { SSTI, necrotizing CAP, bacteremia, } \\
\text { osteomyelitis }\end{array}$ \\
\hline Antibiotic resistance profile & $\begin{array}{l}\text { Multidrug resistant; including } \beta \text {-lactams, } \\
\text { macrolides, TMP-SMX, lincosamides, } \\
\text { tetracyclines, rifampin, quinolones } \\
\text { Growing resistance to glycopeptides also }\end{array}$ & $\begin{array}{l}\text { Resistant to } \beta \text {-lactams. Variable } \\
\text { susceptibility to macrolides, TMP-SMX, } \\
\text { tetracyclines, lincosamydes }\end{array}$ \\
\hline $\begin{array}{l}\text { SCCmec type associated with strains } \\
\text { causing infection }\end{array}$ & I, II and III & IV and V \\
\hline Expression of PVL & Rare & Common \\
\hline
\end{tabular}

HAP: hospital-acquired pneumonia; VAP: ventilator-associated pneumonia; SSTI: skin and soft tissue infection;

CAP: community-acquired pneumonia; TMP-SMX: trimethoprim-sulfamethoxazole; SCCmec: staphylococcal

chromosomal cassette mec; PVL: Panton-Valentine leukocidin; PFGE: pulsed-field gel electrophoresis. 
as clonal complexes (CC): 5, 8, 22, 30 and 45..$^{7,15,16}$ Between 1994 and 2000, surveillance data collected by the CEM/NET initiative identified five predominant pandemic clones (Brazilian, Iberian, Hungarian, pediatric and New York/Japan [NYJ]) within these clonal complexes, and these accounted for nearly $70 \%$ of MRSA isolates worldwide. ${ }^{17}$

The intercontinental spread of MRSA derived from a single ancestral MRSA clone was observed in the USA, UK, Denmark, Switzerland, Egypt and Uganda in the early 1990s. In the late 1990s, further spread was noted when MRSA clones previously isolated from Australia, US and Ireland, were found to have similarities with other MRSA clones collected around the world. ${ }^{13,18}$

Within countries, MRSA clones have disseminated rapidly and efficiently. Diversification and displacement of these clones is similarly rapid. Over a 9-year period in Spain, the common NYJ MRSA clone was displaced by the Brazilian MRSA clone, which was then displaced by the now-predominant EMRSA-16 clone. ${ }^{19}$ Similarly, in Belgium, epidemic MRSA clones belonging to clonal complexes 5, 8, 22, 30 and 45 diversified rapidly, and by 2001, these clonal complexes showed broad geographic distribution in Belgian hospitals, in contrast to earlier survey results. ${ }^{15}$ In Portugal, the predominant Brazilian MRSA clone was replaced in hospitals by two earlier MRSA clones over a 16-year period. ${ }^{16}$

In the USA, HA-MRSA clones are rapidly being replaced by CA-MRSA clones. The extent of this replacement varies between areas, but in one Chicago hospital the proportion of community-associated isolates increased from $24 \%$ to $49 \%$ over 3 years. ${ }^{20}$

\section{MRSA CLONES IN LATIN AMERICA}

Since the first report in 1994 of an autochthonous MRSA clone originating in Brazil, ${ }^{21}$ known as the Brazilian clone, four additional clones circulating in Latin America have been described; the Cordobes, the Pediatric, the Chilean and the NYJ clones. ${ }^{22-24}$ The closely-related Cordobes and Chilean clones are now considered to be a single clone (Cordobes/Chilean). All of these clones circulate extensively in the region (Figure 13-36), and there is evidence supporting the appearance of genetic variants. Several minor clones have also been identified, although these currently occur in restricted geographic areas (Figure $\left.1^{23-36}\right) .^{37}$

The Brazilian clone (ST239-SCCmec type III) has been isolated throughout Brazil and has extended into several other countries, including Argentina, Chile, Colombia, Ecuador, Paraguay, Peru and Uruguay. Several genetic variants have been identified, and together these are termed the Brazilian epidemic clonal complex (BECC). ${ }^{26}$ The pathogenicity of the Brazilian clone derives from several properties, which are present to different degrees in different variants: multidrug resistance, including resistance to -lactams, chloramphenicol, quinolones, lincosamides, erythromy-
Figure 1: MRSA clones circulating in Latin America identified since $2000^{23-36}$

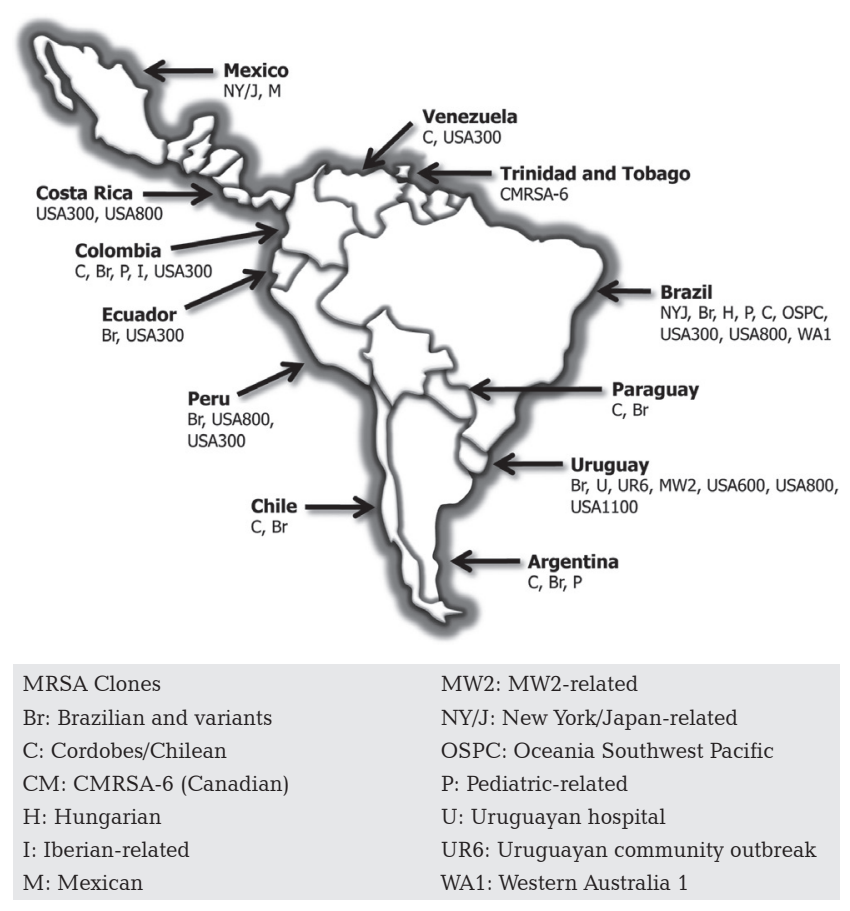

cin, aminoglycosides and trimethoprim-sulfamethoxazole (TMP-SMX); resistance to mupirocin; ${ }^{38}$ production of a protective biofilm; ${ }^{26}$ ability to adhere to, and invade, airway epithelial cells; ${ }^{26}$ and production of toxins, such as enterotoxins and Panton-Valentine leukocidin (PVL). ${ }^{34}$

The Cordobes and Chilean clones (ST5-SCCmec type I) were identified separately in isolates from Argentina ${ }^{23}$ and Chile, ${ }^{22}$ and were later considered to be variants of the same clone. In Argentina, the Cordobes/Chilean clone has rapidly replaced the closely-related Brazilian clone, and it now predominates in several Latin American countries, including Argentina, Chile, Paraguay and Colombia, ${ }^{27,32,35}$ where it is associated with nosocomial outbreaks. The Cordobes/Chilean clone shows a multidrug resistant phenotype, including resistance to erythromycin, although variants are still susceptible to glycopeptides, linezolid, TMP-SMX, rifampin and tetracyclines.

The pediatric (USA800; ST5-SCCmec type IV or SCCmec type VI) and NYJ (USA100; ST5-SCCmec type II) MRSA clones have also spread successfully through Latin America. Variants of the pediatric clone, with heterogeneous and lowlevel resistance to methicillin and resistance to b-lactam antimicrobials, have caused infections in Brazil, Argentina and Colombia, ${ }^{23,30,39}$ and have developed multidrug resistance in some hospitals in Latin America. ${ }^{30}$ The NYJ clone, typically resistant to -lactams, erythromycin, clindamycin and ciprofloxacin, ${ }^{28,33}$ has been detected in Brazil and has completely displaced the Mexican clone in certain Mexican hospitals. ${ }^{24}$

In other Latin American countries, few data are available to describe the spread of individual MRSA clones fully. In a 
recent study, PVL-negative ST5-SCCmec type IV (USA800) and ST8-SCCmec type IV (USA300) MRSA clones were identified in Costa Rica, and a PVL-positive ST5-SCCmec IV clone (USA800) was identified in Peru. ${ }^{29}$ In Trinidad and Tobago, the Canadian MRSA clone, CMRSA-6, was observed in several major hospitals between 2000 and 2001, possibly as a result of tourism. ${ }^{25}$

The first CA-MRSA infections to be reported in Latin America occurred in Brazil in 2003, ${ }^{34}$ where isolates from patients with skin and soft tissue infections (SSTIs) or septic arthritis, were found to harbor SCCmec type IV and PVL. In Uruguay, a large outbreak of CA-MRSA infections in jail inmates occurred, ${ }^{37}$ in which $>1000$ patients were documented with SSTIs and severe forms of pneumonia. This outbreak was caused by a CA-MRSA clone harboring SCCmec type IV and PVL. CA-MRSA clones belonging to CC5, CC8 and CC30 have now been identified in Brazil, Argentina, Colombia and Uruguay, and these are related to clones previously observed in the USA and Australia. In addition, community-acquired infections due to MRSA have been reported in Peru, ${ }^{40}$ Venezuela ${ }^{41}$ and Chile, ${ }^{42,43}$ although some of these cases were in people returning from cities in Uruguay or Brazil where a higher incidence of community-acquired MRSA infections occurred. In a recent study in four South American countries (Colombia, Ecuador, Peru and Venezuela), Arias and colleagues found a new CA-MRSA USA300 variant in the Andean region. ${ }^{36}$

\section{CLINICAL IMPLICATIONS OF MRSA CLONES}

\section{Virulence and antibiotic resistance of MRSA clones}

MRSA strains cause a variety of infections, ranging in severity from cutaneous abscesses, to life-threatening necrotizing fasciitis and necrotizing pneumonia. The severity of disease is usually directly related to the production by MRSA of specific virulence factors, ${ }^{44}$ such as toxins or protective biofilms, whereas the spread of MRSA depends partly on the ability of individual clones to acquire resistance to antibacterial agents.

\section{Panton-Valentine Leukocidin Production}

PVL, a major virulence factor for MRSA, is a secreted toxin that causes tissue necrosis and damage to immune cells. The gene encoding PVL, pvl, encodes two secreted subunits, designated LukS-PV and LukF-PV, which assemble together in the membranes of white blood cells, monocytes and macrophages to form pores through which cell contents leak. ${ }^{45}$ PVL-producing MRSA strains have been associated with SSTIs and a severe form of necrotizing pneumonia. ${ }^{46,47}$ In a US study of 422 patients with community-acquired SSTIs, 59\% of isolates were identified as MRSA (mainly clone USA300), and $98 \%$ of these expressed PVL, ${ }^{48}$ consistent with other studies. ${ }^{49}$ The presence of PVL has now been demonstrated in both adult and pediatric MRSA infections. ${ }^{49,50}$
Acquisition of $p v l$ by CA-MRSA clones marked a dramatic change in the epidemiology of MRSA infections, both in the community and, more recently, in hospitals. ${ }^{51-53}$ Several studies have reported the acquisition of $p v l$ by MRSA clones circulating in Latin America. ${ }^{34,54,55}$ In Rio de Janeiro, for example, BECC isolates were shown to be positive for $p v l$, possibly following horizontal transfer from a reservoir of PVL-positive MSSA isolates. ${ }^{54}$ In Argentina, 94\% of isolates of the predominant CA-MRSA clone ST5 were found to harbor $p v l .{ }^{35}$

\section{Biofilm production by MRSA strains}

$S$. aureus strains routinely produce and become encased in biofilm, providing them with protection against both host defenses and antimicrobial drugs. Biofilm production is mediated by the ica operon, and this property is present in most MRSA and MSSA strains, although evidence suggests that certain MRSA clones have an enhanced ability in this regard. In 2005, the predominant variant of the BECC MRSA clone was reported to be more effective in generating biofilm and in adhering to, and invading, airway epithelial cells, than MSSA or sporadic MRSA clones. ${ }^{26}$ In a recent Brazilian study of MRSA isolates from hospital-acquired and communityacquired MRSA infections, all 19 MRSA strains (14 Brazilian clone; $5 \mathrm{NYJ}$ clone) were found to produce biofilm. ${ }^{56}$

\section{Resistance in MRSA clones}

MRSA, and in some countries vancomycin-resistant enterococci, are the most frequent antibiotic-resistant gram-positive bacteria responsible for nosocomial infections and, in the case of MRSA, community infections. ${ }^{57}$

HA-MRSA strains are often multidrug resistant conferred by SCCmec types II and III, ${ }^{58}$ and can express resistance to fluoroquinolones, macrolides, aminoglycosides, tetracyclines and rifampin. Multidrug resistant MRSA strains can also develop reduced susceptibility or resistance to vancomycin and teicoplanin, as well as to newer antimicrobials such as quinupristin-dalfopristin, linezolid, daptomycin and tigecycline, although these occurrences are limited to a few isolated cases. S. aureus with reduced susceptibility to vancomycin, or vancomycin-intermediate $S$. aureus (VISA), usually develop this limited susceptibility by changing the thickness of their cell wall. Consequently, vancomycin becomes trapped in the outer wall and has reduced access to its target in the cytoplasmic membrane. S. aureus can also develop full resistance to vancomycin (VRSA), which is thought to be linked to the acquisition of the resistance gene van $\mathrm{A}$ from vancomycin-resistant enterococci..$^{59,60}$

Clindamycin is a commonly used antibiotic for the treatment of infections caused by MSSA and MRSA, such as SSTIs. However, S. aureus clones have emerged with inducible resistance to clindamycin following a ribosomal target modification. Resistance to clindamycin is induced by macrolides, and can be detected following erythromycin- 
mediated induction using the approximation test. ${ }^{61}$ Studies based in the USA report the overall prevalence of inducible clindamycin resistance in S. aureus isolates as $52 \%(50 \%$ of MRSA and $60 \%$ of MSSA isolates), ${ }^{62}$ although this may change, since clonal shift has been shown to affect inducible clindamycin resistance. ${ }^{63}$

In Latin America, MRSA clones are genetically diverse, but often share in common genes that encode for multidrug resistance to -lactams, erythromycin, chloramphenicol and clindamycin, and variable resistance to rifampin, fluorquinolones and TMP-SMX. Three classes of antimicrobials, including glycopeptides, oxazolidinones and the new tetracycline derivative tigecycline, are uniformly active against these clonal variants in the region. The Brazilian clone is sometimes only sensitive to vancomycin, tigecycline, linezolid and daptomycin. Although there are reports indicating heteroresistance to vancomycin in the Brazilian clone, there is currently little evidence to suggest that VISA and/or VRSA are a significant issue in Latin America. However, it will be important to monitor the situation across the region in order to identify any changes to the pattern of antimicrobial resistance as early as possible. The Cordobes/Chilean and NYJ clones, and in some places also the pediatric clone, are multidrug resistant. Usually, the Cordobes/Chilean clone is susceptible to glycopeptides, linezolid, TMP-SMX, minocycline and rifampin, while the NYJ clone is sensitive to glycopeptides, linezolid, TMP-SMX, rifampin and gentamicin. Careful use of antibiotics is an important strategy for limiting antimicrobial resistance. For example, high level resistance of the Brazilian clone to mupirocin has been reported in Brazilian hospitals, ${ }^{38}$ but recent evidence suggests that resistance can be reduced by controlling mupirocin use. ${ }^{64}$

\section{MRSA clones in hospital and community settings}

Isolates from nosocomial MRSA infections, typically collected $>72$ hours following hospital admission, usually, but not always, contain MRSA clones harboring SCCmec type I, II or III (Table 1). These clones produce bacteremia, pneumonia or urinary tract infections more frequently than they cause SSTI. ${ }^{31,65}$ Nosocomial MRSA is usually reported as an adult pathogen, but has also been associated with infections in pediatric and neonatal intensive care units. ${ }^{24,66}$

Isolates from community-acquired MRSA infections, typically collected $<72$ hours after admission to hospital, usually contain CA-MRSA clones that harbor SCCmec type IV and produce SSTI (Table 1). These clones tend to be sensitive to most antimicrobials, with the exception of erythromycin and ciprofloxacin, and commonly produce the virulence factor PVL.

\section{RECOMBINATION BETWEEN COMMUNITY AND HOSPITAL MRSA CLONES}

There is growing evidence that virulence genes can be transferred between HA- and CA-MRSA strains. For example,
SCCmec type IV traits, usually associated with CA-MRSA, have been observed in nosocomial MRSA strains with susceptibility to four or more antimicrobials ${ }^{67}$ suggesting that CA-MRSA clones have been introduced into the hospital and are now circulating as nosocomial pathogens. Similarly, biofilm- and enterotoxin-producing, non-multidrug resistant MRSA that displayed PFGE patterns similar to USA800 caused severe nosocomial infections in two Brazilian hospitals in 2007. ${ }^{68}$ Clones originating in hospitals have also been found to be spreading in the community. In Brazil, MRSA strains isolated from the nasopharyngeal passages of children recently admitted to hospital between 2000 and 2001, were found to be multidrug resistant and harboring SCCmec type III. ${ }^{69}$

\section{INFLUENCE OF MRSA CLONES ON DISEASE AND CLINICAL PRACTICE}

\section{MRSA clones and nasal carriers}

In a recent study, over $7 \%$ of hospital admissions were estimated to be nasal carriers of MRSA ${ }^{70}$ with the nares potentially serving as a colonizing point and reservoir for further infection. While most carriers of $S$. aureus are thought to be colonized by a single $S$. aureus clone, approximately $7 \%$ of carriers in one study were found to be 'discordant' carriers, colonized by more than one strain, and in some cases carriers were colonized by MRSA and MSSA strains simultaneously. ${ }^{71}$ In discordant carriers, the presence of multiple strains of $S$. aureus presents the opportunity for horizontal genetic information exchange between strains. ${ }^{71}$

MRSA clones isolated from the anterior nares can be varied, but are often community clones. ${ }^{70,72}$ In a homeless population study in the USA, ${ }^{72} 6.2 \%$ of individuals carried nasal MRSA clones, most of which were the CA-MRSA clones USA300 and USA1000. In children within Brazilian day-care centers, several MRSA clonal types, including SCCmec types IIIA, IV and V, have been identified. ${ }^{73}$

Studies suggest that nasal carriers of $S$. aureus may develop infection at a further site with the same clone. For example, in one study, $>82 \%$ patients who developed bacteremia carried identical S. aureus clones in the anterior nares. ${ }^{74}$ Furthermore, clones present in the anterior nares were shown to appear in bacteremia infections in the same patients up to 14 months later. In another study, $>67 \%$ patients with clinical MRSA infections were found to carry MRSA in the nares, and nasal carriers of MRSA carried an enhanced risk of MRSA infections at other sites compared with the remainder of the population. ${ }^{75}$ Healthcare workers are also at risk of becoming carriers with the same clones that are present in hospitalized patients, ${ }^{76}$ and risk transmitting this pathogen to close household contacts. ${ }^{77}$ 


\section{MRSA clones and skin and soft tissue infections}

MRSA is a prevalent and widespread cause of SSTIs. In a US study, MRSA was the most common pathogen-causing SSTI identified in emergency departments, with a prevalence of $59 \%$ overall. $^{48}$ Of the MRSA isolates, $72 \%$ were a single strain, USA300-0114, which harbored SCCmec type IV and was positive for PVL. These isolates were associated with superficial infections and abscesses, and with deeper infections and osteomyelitis. ${ }^{48}$ MRSA was also the most common pathogen associated with SSTIs requiring surgical debridement in a 7 -year study in Houston. ${ }^{78}$

While most of the MRSA strains causing SSTIs are of community origin worldwide, differences in clonality and resistance pattern of MRSA strains between close geographic areas have been reported. In California, for example, CA-MRSA accounted for $93 \%$ of SSTIs in San Francisco General Hospital between 2000 and 2002, but only for 69\% in Stanford University Hospital; by 2002, PVL-positive ST8SCCmec type IV (USA300) was the predominant strain in San Francisco, showing sensitivity to most antimicrobials, whereas the multidrug resistant clone ST5-SCCmec type II (USA100) was the predominant clone causing SSTI at Stanford University Hospital. ${ }^{79}$ This study highlights the importance of local surveillance knowledge, even at the level of individual hospitals within the same vicinity, when choosing antimicrobial therapy for community-based SSTIs.

In Latin America, limited data are available to draw conclusions on the associations of clones with SSTIs. In the first report of community-acquired infections in the region, Ribeiro and colleagues ${ }^{34}$ observed PVL-positive SCCmec type IV CA-MRSA strains in two patients with SSTI and one with septic arthritis, which corresponded to the Oceania Southwest Pacific clone (OSPC) from Australia, and shared genotypic characteristics with CA-MRSA strains in the USA and in Europe. A surveillance study conducted in 2005 in Cordoba, Argentina, found that $90 \%$ of infections caused by CA-MRSA were SSTIs, and 89\% were caused by CC5:ST5 SCCmec type IVa-c strains, ${ }^{35}$ whereas in Costa Rica, clones CC5:ST5 SCCmec type IV (USA 800) and CC8:SLV8 SCCmec type IV (USA 300) were found in community isolates from patients with SSTI, both of which were negative for PVL. ${ }^{29}$

\section{MRSA clones and bacteremia}

Data from the SENTRY Antimicrobial Surveillance Program in Argentina, Brazil, Chile, Colombia, Venezuela and Uruguay, have shown that MRSA is a common cause of nosocomial bacteremia, with a prevalence of $21.6 \%$ among bloodstream infection isolates. ${ }^{80}$ MRSA nosocomial bacteremia causes severe complications, including infectious endocarditis, ${ }^{81}$ and is associated with elevated morbidity and mortality. ${ }^{82}$ The selection of antibiotic treatment and the duration of treatment for MRSA nosocomial bacteremia is one of the most controversial issues in medicine..$^{81,82}$
Bacteremia can be caused by both CA- and HA-MRSA clones, and the severity of disease is dependent on clonal type. In a recent study, ${ }^{83}$ SCCmec type II HA-MRSA clones were found to cause higher mortality than SCCmec type IVa CA-MRSA clones, whereas SCCmec type IVa clones caused greater metastatic infection. Also, while the majority of community-acquired and hospital-acquired bacteremias were caused by SCCmec type IVa and SCCmec type II MRSA clones, respectively, SCCmec type IVa was present in some hospital-acquired bacteremias, and SCCmec type II was present in some community-acquired bacteremias. ${ }^{83}$

\section{MRSA clones and infectious endocarditis}

MRSA is a common pathogen in infectious endocarditis (IE), with prevalence of over one third in some countries, including Brazil (37.5\%) and the USA (37.2\%). ${ }^{81}$ S. aureus IE is usually acquired outside hospital, but predominates as a healthcare-associated infection, which accounts for $54 \%$ of cases in Brazil. ${ }^{81}$ The first reported case of IE in Brazil caused by CA-MRSA was published in $2008,{ }^{84}$ and was attributed to MRSA harboring SCCmec type IV and positive for PVL. In Korea, a PVL-negative CA-MRSA clone ST72 SCCmec IVa has been identified as the cause of IE infections in previously healthy individuals with no reported risk factors for IE. ${ }^{85}$

\section{CONCLUSION: CURRENT CHALLENGES AND FUTURE DIRECTIONS}

Molecular epidemiological studies have highlighted the continuing global evolution and spread of MRSA clones with increased resistance to antimicrobial drugs and enhanced virulence. The factors contributing to the dissemination of MRSA clones are only partially understood, but are thought to include the migration of human populations, ineffective methods to control transmission of MRSA from infected patients, and treatment strategies, including the inappropriate use and choice of antibiotics. ${ }^{86,87}$ In hospitals, patients already carrying MRSA when admitted are at greater risk of developing an infection derived from the colonized bacteria, or of transmitting MRSA to other patients.

In Latin America, pandemic clones are commonplace in hospitals across the region, and community-associated infections are growing in number. ${ }^{1}$ Clones circulating in the region show genetic diversity, although common genes encoding multidrug resistance to antimicrobials are expressed. Enhanced pathogenic properties, including production of biofilms and production of enterotoxins, have been described for certain clones, and the ability of nosocomial and community clones to interchange genetic material has also been identified.

However, surveillance data for specific MRSA clones within the region is limited, ${ }^{1}$ with existing data biased towards more developed countries and sophisticated research 
centers. Paradoxically, countries showing the highest prevalence of nosocomial MRSA infections, such as Peru, often provide very little information with regard to molecular epidemiology and clinical outcome. Regional surveillance programs, using central reference laboratories and integrating information from health centers with different complexities, are required if we are to understand more fully the developing pattern of MRSA infections across Latin America, and to design better treatment and prevention strategies.

\section{IMPLICATIONS FOR CLINICAL PRACTICE}

- The characteristics of different MRSA clones are associated with clinical presentation and outcomes.

- Clonal dissemination is rapid for both CAMRSA and HA-MRSA, and recombination between $\mathrm{CA}$ and HA clones is taking place.

- Regional molecular epidemiology programs are required to understand more fully the developing pattern of MRSA infections across Latin America.

- More accurate information on the precise distribution of clones in the region may help clinicians in understanding the risk of infection by certain clones and in choosing appropriate empiric antimicrobial therapy.

\section{ACKNOWLEDGEMENTS}

\section{Financial support}

Pfizer Inc., New York, NY, USA, provided support for meetings of the Latin American Working Group on Gram Positive Resistance. Pfizer Inc. had no involvement in the collection, analysis and interpretation of data, in the writing of the manuscripts, or in the decision to submit the articles for publication.

\section{Manuscript preparation}

The support provided by Choice Pharma (Hitchin, UK), funded by Pfizer Inc., consisted of manuscript formatting and writing assistance.

\section{DISCLOSURES}

E. Rodríguez-Noriega: Advisory Board member for Pfizer; consultant for Pfizer, Wyeth, Johnson \& Johnson and Novartis; received research grants from Pfizer, Wyeth, Johnson \& Johnson, Schering-Plough and Cerexa.

C. Seas: Advisory Board member and consultant for Pfizer; received research funding from Therevance, Cerexa, Schering-Plough and Avexa.

\section{REFERENCES}

1. Guzmán-Blanco M, Mejía C, Isturiz R et al. Epidemiology of methicillin-resistant Staphylococcus aureus (MRSA) in Latin America. Int J Antimicrob Agents. 2009; 34(4):304-8.

2. Rodríguez-Noriega E, Seas C, Guzmán-Blanco M et al. Evolution of methicillin-resistant Staphylococcus aureus clones in Latin America. Int J Infect Dis. 2010; 14:e560-6.

3. Ito T, Ma XX, Takeuchi F et al. Novel type V staphylococcal cassette chromosome mec driven by a novel cassette chromosome recombinase, ccrC. Antimicrob Agents Chemother. 2004; 48(7):2637-51.

4. Jevons MP, Coe AW, Parker MT. Methicillin resistance in staphylococci. Lancet. 1963; 1(7287):904-7.

5. Crisostomo MI, Westh H, Tomasz A et al. The evolution of methicillin resistance in Staphylococcus aureus: similarity of genetic backgrounds in historically early methicillin-susceptible and -resistant isolates and contemporary epidemic clones. Proc Natl Acad Sci U S A. 2001; 98(17):9865-70.

6. Deurenberg RH, Stobberingh EE. The molecular evolution of hospital- and community-associated methicillin-resistant Staphylococcus aureus. Curr Mol Med. 2009; 9(2):100-15.

7. Deurenberg RH, Stobberingh EE. The evolution of Staphylococcus aureus. Infect Genet Evol. 2008; 8(6):747-63.

8. Donnio PY, Fevrier F, Bifani P et al. Molecular and epidemiological evidence for spread of multiresistant methicillin-susceptible Staphylococcus aureus strains in hospitals. Antimicrob Agents Chemother. 2007; 51(12):4342-50.

9. Cookson BD, Robinson DA, Monk AB et al. Evaluation of molecular typing methods in characterizing a European collection of epidemic methicillin-resistant Staphylococcus aureus strains: the HARMONY collection. J Clin Microbiol. 2007; 45(6):1830-7.

10. Naimi TS, LeDell KH, Como-Sabetti K et al. Comparison of community- and health care-associated methicillin-resistant Staphylococcus aureus infection. JAMA 2003; 290(22):2976-84.

11. Gorwitz RJ. Understanding the success of methicillin-resistant Staphylococcus aureus strains causing epidemic disease in the community. J Infect Dis. 2008; 197(2):179-82.

12. Flynn N, Cohen SH. The continuing saga of MRSA. J Infect Dis. 2008; 197(9):1217-9.

13. Ayliffe GA. The progressive intercontinental spread of methicillin-resistant Staphylococcus aureus. Clin Infect Dis. 1997; 24(Suppl 1):S74-9.

14. Robinson DA, Enright MC. Multilocus sequence typing and the evolution of methicillin-resistant Staphylococcus aureus. Clin Microbiol Infect. 2004; 10(2):92-7.

15. Denis O, Deplano A, Nonhoff C et al. National surveillance of methicillin-resistant Staphylococcus aureus in Belgian hospitals indicates rapid diversification of epidemic clones. Antimicrob Agents Chemother. 2004; 48(9):3625-9.

16. Aires-de-Sousa M, Correia B, de Lencastre H. Changing patterns in frequency of recovery of five methicillin-resistant Staphylococcus aureus clones in Portuguese hospitals: surveillance over a 16-year period. J Clin Microbiol. 2008; 46(9):2912-7.

17. Oliveira DC, Tomasz A, de Lencastre H. Secrets of success of a human pathogen: molecular evolution of pandemic clones of meticillin-resistant Staphylococcus aureus. Lancet Infect Dis. 2002; 2(3):180-9.

18. Musser JM, Kapur V. Clonal analysis of methicillin-resistant Staphylococcus aureus strains from intercontinental sources: association of the mec gene with divergent phylogenetic lineages implies dissemination by horizontal transfer and recombination. J Clin Microbiol. 1992; 30(8):2058-63. 
19. Potel C, Alvarez M, Alvarez P et al. Evolution, antimicrobial susceptibility and assignment to international clones of methicillinresistant Staphylococcus aureus isolated over a 9-year period in two Spanish hospitals. Clin Microbiol Infect. 2007; 13(7):728-30.

20. Popovich KJ, Weinstein RA, Hota B. Are community-associated methicillin-resistant Staphylococcus aureus (MRSA) strains replacing traditional nosocomial MRSA strains? Clin Infect Dis. 2008; 46(6):787-94.

21. Sader HS, Pignatari AC, Hollis RJ, Jones RN. Evaluation of interhospital spread of methicillin-resistant Staphylococcus aureus in Sao Paulo, Brazil, using pulsed-field gel electrophoresis of chromosomal DNA. Infect Control Hosp Epidemiol. 1994; 15(5):320-3.

22. Aires de Sousa M, Miragaia M, Sanches IS et al. Three-year assessment of methicillin-resistant Staphylococcus aureus clones in Latin America from 1996 to 1998. J Clin Microbiol. 2001; 39(6):2197-205.

23. Sola C, Gribaudo G, Vindel A et al. Identification of a novel methicillin-resistant Staphylococcus aureus epidemic clone in Cordoba, Argentina, involved in nosocomial infections. J Clin Microbiol. 2002; 40(4):1427-35.

24. Velazquez-Meza ME, Aires de Sousa M, Echaniz-Aviles G et al. Surveillance of methicillin-resistant Staphylococcus aureus in a pediatric hospital in Mexico City during a 7-year period (1997 to 2003): clonal evolution and impact of infection control. J Clin Microbiol. 2004; 42(8):3877-80.

25. Akpaka PE, Kissoon S, Rutherford C et al. Molecular epidemiology of methicillin-resistant Staphylococcus aureus isolates from regional hospitals in Trinidad and Tobago. Int J Infect Dis. 2007; 11(6):544-8.

26. Amaral MM, Coelho LR, Flores RP et al. The predominant variant of the Brazilian epidemic clonal complex of methicillin-resistant Staphylococcus aureus has an enhanced ability to produce biofilm and to adhere to and invade airway epithelial cells. J Infect Dis. 2005; 192(5):801-10.

27. Cruz C, Moreno J, Renzoni A et al. Tracking methicillin-resistant Staphylococcus aureus clones in Colombian hospitals over 7 years (1996-2003): emergence of a new dominant clone. Int J Antimicrob Agents. 2005; 26(6):457-62.

28. Echaniz-Aviles G, Velazquez-Meza ME, Aires-de-Sousa M et al. Molecular characterisation of a dominant methicillin-resistant Staphylococcus aureus (MRSA) clone in a Mexican hospital (1999-2003). Clin Microbiol Infect. 2006; 12(1):22-8.

29. Goering RV, Shawar RM, Scangarella NE et al. Molecular epidemiology of methicillin-resistant and methicillin-susceptible Staphylococcus aureus isolates from global clinical trials. J Clin Microbiol. 2008; 46(9):2842-7.

30. Gomes AR, Sanches IS, Aires de Sousa M et al. Molecular epidemiology of methicillin-resistant Staphylococcus aureus in Colombian hospitals: dominance of a single unique multidrug-resistant clone. Microb Drug Resist. 2001; 7(1):23-32.

31. Ramirez Barba EJ, Rosenthal VD, Higuera F et al. Device-associated nosocomial infection rates in intensive care units in four Mexican public hospitals. Am J Infect Control. 2006; 34(4):244-7.

32. Mayor L, Ortellado J, Menacho C et al. Molecular characterization of methicillin-resistant Staphylococcus aureus isolates collected in Asuncion, Paraguay. J Clin Microbiol. 2007; 45(7):2298-300.

33. Melo MC, Silva-Carvalho MC, Ferreira RL et al. Detection and molecular characterization of a gentamicin-susceptible, methicillin-resistant Staphylococcus aureus (MRSA) clone in Rio de Janeiro that resembles the New York/Japanese clone. J Hosp Infect. 2004; 58(4):276-85.
34. Ribeiro A, Dias C, Silva-Carvalho MC et al. First report of infection with community-acquired methicillin-resistant Staphylococcus aureus in South America. J Clin Microbiol. 2005; 43(4):1985-8.

35. Sola C, Saka HA, Vindel A, Bocco JL. Emergence and dissemination of a community-associated methicillin-resistant Panton-Valentine leucocidin-positive Staphylococcus aureus clone sharing the sequence type 5 lineage with the most prevalent nosocomial clone in the same region of Argentina. J Clin Microbiol. 2008; 46(5):1826-31.

36. Reyes J, Rincon S, Diaz L et al. Dissemination of methicillinresistant Staphylococcus aureus USA300 sequence type 8 lineage in Latin America. Clin Infect Dis. 2009; 49(12):1861-7.

37. Ma XX, Galiana A, Pedreira W et al. Community-acquired methicillin-resistant Staphylococcus aureus, Uruguay. Emerg Infect Dis. 2005; 11(6):973-6.

38. Ramos RL, Teixeira LA, Ormonde LR et al. Emergence of mupirocin resistance in multiresistant Staphylococcus aureus clinical isolates belonging to Brazilian epidemic clone III::B:A. J Med Microbiol. 1999; 48(3):303-7.

39. Corso A, Santos Sanches I, Aires de Sousa M et al. Spread of a methicillin-resistant and multiresistant epidemic clone of Staphylococcus aureus in Argentina. Microb Drug Resist. 1998; 4(4):277-88.

40. Seas C, Hernandez K, Ramos R et al. Oxacillin-resistant and multidrug-resistant Staphylococcus aureus in Lima, Peru. Infect Control Hosp Epidemiol. 2006; 27(2):198-200.

41. Pfizer Venezuela S.A. Venezuelan program of surveillance of bacterial resistance to antibiotics; 1998-2006. (http://www. provenra.org) Accessed 19 May 2010.

42. Aylwin M, Serri M, Gracía P et al. Staphylococcus aureus meticilino resistente asociado a la comunidad en Chile (A-C021). Abstract presented at the XXIV Chilean Conference of Infectious Diseases. Pucón, Chile, 2007.

43. Noríega LM, Gonzalez P, Hormazabal JC et al. Community acquired infections with methicillin resistant strains of Staphylococcus aureus: report of five cases. Rev Med Chil. 2008; 136(7):885-91.

44. Diep BA, Carleton HA, Chang RF et al. Roles of 34 virulence genes in the evolution of hospital- and community-associated strains of methicillin-resistant Staphylococcus aureus. J Infect Dis. 2006; 193(11):1495-503.

45. Diep BA, Palazzolo-Ballance AM, Tattevin P et al. Contribution of Panton-Valentine leukocidin in community-associated methicillin-resistant Staphylococcus aureus pathogenesis. PLoS One. 2008; 3(9):e3198.

46. Diep BA, Sensabaugh GF, Somboona NS et al. Widespread skin and soft-tissue infections due to two methicillin-resistant Staphylococcus aureus strains harboring the genes for Panton-Valentine leucocidin. J Clin Microbiol. 2004; 42(5):2080-4.

47. Francis JS, Doherty MC, Lopatin U et al. Severe communityonset pneumonia in healthy adults caused by methicillin-resistant Staphylococcus aureus carrying the Panton-Valentine leukocidin genes. Clin Infect Dis. 2005; 40(1):100-7.

48. Moran GJ, Krishnadasan A, Gorwitz RJ et al. Methicillin-resistant $S$. aureus infections among patients in the emergency department. N Engl J Med. 2006; 355(7):666-74.

49. Limbago B, Fosheim GE, Schoonover V et al. Characterization of methicillin-resistant Staphylococcus aureus isolates collected in 2005 and 2006 from patients with invasive disease: a population-based analysis. J Clin Microbiol. 2009; 47(5):1344-51. 
50. McCaskill ML, Mason EO, Jr., Kaplan SL et al. Increase of the USA300 clone among community-acquired methicillinsusceptible Staphylococcus aureus causing invasive infections. Pediatr Infect Dis J. 2007; 26(12):1122-7.

51. Lina G, Piemont Y, Godail-Gamot F et al. Involvement of Panton-Valentine leukocidin-producing Staphylococcus aureus in primary skin infections and pneumonia. Clin Infect Dis. 1999; 29(5):1128-32.

52. Gillet Y, Issartel B, Vanhems P et al. Association between Staphylococcus aureus strains carrying gene for Panton-Valentine leukocidin and highly lethal necrotising pneumonia in young immunocompetent patients. Lancet. 2002; 359(9308):753-9.

53. Tristan A, Bes M, Meugnier H et al. Global distribution of Panton-Valentine leukocidin-positive methicillin-resistant Staphylococcus aureus, 2006. Emerg Infect Dis. 2007; 13(4):594-600.

54. Vivoni AM, Diep BA, de Gouveia Magalhaes AC et al. Clonal composition of Staphylococcus aureus isolates at a Brazilian university hospital: identification of international circulating lineages. J Clin Microbiol. 2006; 44(5):1686-91.

55. Sola C, Saka HA, Vindel A, Bocco JL. High frequency of Panton-Valentine leukocidin genes in invasive methicillin-susceptible Staphylococcus aureus strains and the relationship with methicillin-resistant Staphylococcus aureus in Cordoba, Argentina. Eur J Clin Microbiol Infect Dis. 2007; 26(4):281-6.

56. Souza RR, Coelho LR, Botelho AM et al. Biofilm formation and prevalence of lukF-pv, seb, sec and tst genes among hospital- and community-acquired isolates of some international methicillin-resistant Staphylococcus aureus lineages. Clin Microbiol Infect. 2009; 15(2):203-7.

57. Rice LB. Antimicrobial resistance in gram-positive bacteria. Am J Med. 2006; 119(6 Suppl 1):S11-9; discussion S62-70.

58. Deurenberg RH, Vink C, Kalenic S et al. The molecular evolution of methicillin-resistant Staphylococcus aureus. Clin Microbiol Infect. 2007; 13(3):222-35.

59. Chang S, Sievert DM, Hageman JC et al. Infection with vancomycin-resistant Staphylococcus aureus containing the vanA resistance gene. N Engl J Med. 2003; 348(14):1342-7.

60. Tenover FC, Moellering RC, Jr. The rationale for revising the Clinical and Laboratory Standards Institute vancomycin minimal inhibitory concentration interpretive criteria for Staphylococcus aureus. Clin Infect Dis. 2007; 44(9):1208-15.

61. Fiebelkorn KR, Crawford SA, McElmeel ML, Jorgensen JH. Practical disk diffusion method for detection of inducible clindamycin resistance in Staphylococcus aureus and coagulasenegative staphylococci. J Clin Microbiol. 2003; 41(10):4740-4.

62. Patel M, Waites KB, Moser SA et al. Prevalence of inducible clindamycin resistance among community- and hospital-associated Staphylococcus aureus isolates. J Clin Microbiol. 2006; 44(7):2481-4

63. Chavez-Bueno S, Bozdogan B, Katz K et al. Inducible clindamycin resistance and molecular epidemiologic trends of pediatric community-acquired methicillin-resistant Staphylococcus aureus in Dallas, Texas. Antimicrob Agents Chemother. 2005; 49(6):2283-8.

64. Vivoni AM, Santos KR, de-Oliveira MP et al. Mupirocin for controlling methicillin-resistant Staphylococcus aureus: lessons from a decade of use at a university hospital. Infect Control Hosp Epidemiol. 2005; 26(7):662-7.

65. Safdar N, Bradley EA. The risk of infection after nasal colonization with Staphylococcus aureus. Am J Med. 2008; 121(4):310-5.

66. Saiman L, Cronquist A, Wu F et al. An outbreak of methicillin-resistant Staphylococcus aureus in a neonatal intensive care unit. Infect Control Hosp Epidemiol. 2003; 24(5):317-21.
67. de A Trindade P, Pacheco RL, Costa SF et al. Prevalence of SCCmec type IV in nosocomial bloodstream isolates of methicillin-resistant Staphylococcus aureus. J Clin Microbiol. 2005; 43(7):3435-7.

68. de Miranda OP, Silva-Carvalho MC, Ribeiro A et al. Emergence in Brazil of methicillin-resistant Staphylococcus aureus isolates carrying SCCmecIV that are related genetically to the USA800 clone. Clin Microbiol Infect. 2007; 13(12):1165-72.

69. Lamaro-Cardoso J, Castanheira M, de Oliveira RM et al. Carriage of methicillin-resistant Staphylococcus aureus in children in Brazil. Diagn Microbiol Infect Dis. 2007; 57(4):467-70.

70. Hidron AI, Kourbatova EV, Halvosa JS et al. Risk factors for colonization with methicillin-resistant Staphylococcus aureus (MRSA) in patients admitted to an urban hospital: emergence of community-associated MRSA nasal carriage. Clin Infect Dis. 2005; 41(2):159-66.

71. Cespedes C, Said-Salim B, Miller M et al. The clonality of Staphylococcus aureus nasal carriage. J Infect Dis. 2005; 191(3):444-52.

72. Pan ES, Diep BA, Charlebois ED et al. Population dynamics of nasal strains of methicillin-resistant Staphylococcus aureusand their relation to community-associated disease activity. J Infect Dis. 2005; 192(5):811-8.

73. Lamaro-Cardoso J, de Lencastre H, Kipnis A et al. Molecular epidemiology and risk factors for nasal carriage of Staphylococcus aureus and methicillin-resistant $S$. aureus in infants attending day-care centers in Brazil. J Clin Microbiol. 2009; 47(12):3991-7.

74. von Eiff C, Becker K, Machka K et al. Nasal carriage as a source of Staphylococcus aureus bacteremia. Study Group. N Engl J Med. 2001; 344(1):11-6.

75. Robicsek A, Suseno M, Beaumont JL et al. Prediction of methicillin-resistant Staphylococcus aureus involvement in disease sites by concomitant nasal sampling. J Clin Microbiol. 2008; 46(2):588-92.

76. Cespedes C, Miller M, Quagliarello B et al. Differences between Staphylococcus aureus isolates from medical and nonmedical hospital personnel. J Clin Microbiol. 2002; 40(7):2594-7.

77. Eveillard M, Martin Y, Hidri N et al. Carriage of methicillinresistant Staphylococcus aureus among hospital employees: prevalence, duration, and transmission to households. Infect Control Hosp Epidemiol. 2004; 25(2):114-20.

78. Awad SS, Elhabash SI, Lee L et al. Increasing incidence of methicillin-resistant Staphylococcus aureus skin and soft-tissue infections: reconsideration of empiric antimicrobial therapy. Am J Surg. 2007; 194(5):606-10.

79. Bhattacharya D, Carleton $\mathrm{H}$, Tsai CJ et al. Differences in clinical and molecular characteristics of skin and soft tissue methicillin-resistant Staphylococcus aureus isolates between two hospitals in Northern California. J Clin Microbiol. 2007; 45(6):1798-803.

80. Biedenbach DJ, Moet GJ, Jones RN. Occurrence and antimicrobial resistance pattern comparisons among bloodstream infection isolates from the SENTRY Antimicrobial Surveillance Program (1997-2002). Diagn Microbiol Infect Dis. 2004; 50(1):59-69.

81. Fowler VG, Jr., Miro JM, Hoen B et al. Staphylococcus aureus endocarditis: a consequence of medical progress. JAMA. 2005; 293(24):3012-21.

82. Corey GR. Staphylococcus aureus bloodstream infections: definitions and treatment. Clin Infect Dis. 2009; 48 (Suppl 4):S254-9. 
83. Ganga R, Riederer K, Sharma M et al. Role of SCCmec type in outcome of Staphylococcus aureus bacteremia in a single medical center. J Clin Microbiol. 2009; 47(3):590-5.

84. Fortes CQ, Espanha CA, Bustorff FP et al. First reported case of infective endocarditis caused by community-acquired methicillin-resistant Staphylococcus aureus not associated with healthcare contact in Brazil. Braz J Infect Dis. 2008; 12(6):541-3.

85. Lee SY, Kim JY, Kim JH et al. A case of primary infective endocarditis caused by community-associated methicillin-resistant Staphylococcus aureus in a healthy individual and colonization in the family. Yonsei Med J. 2009; 50(1):152-5.
86. Aires de Sousa M, Sanches IS, Ferro ML et al. Intercontinental spread of a multidrug-resistant methicillin-resistant Staphylococcus aureus clone. J Clin Microbiol. 1998; 36(9):2590-6.

87. Shopsin B, Mathema B, Zhao X et al. Resistance rather than virulence selects for the clonal spread of methicillin-resistant Staphylococcus aureus: implications for MRSA transmission. Microb Drug Resist. 2000; 6(3):239-44. 